\title{
LOW-ORDER DIVERGENCE-FREE APPROXIMATIONS FOR THE STOKES PROBLEM ON WORSEY-FARIN AND POWELL-SABIN SPLITS
}

\author{
MAURICE FABIEN ${ }^{\dagger}$, JOHNNY GUZMÁN $^{\dagger}$, MICHAEL NEILAN $^{\ddagger}$, AND AHMED ZYTOON
}

\begin{abstract}
We derive low-order, inf-sup stable and divergence-free finite element approximations for the Stokes problem using Worsey-Farin splits in three dimensions and Powell-Sabin splits in two dimensions. The velocity space simply consists of continuous, piecewise linear polynomials, where as the pressure space is a subspace of piecewise constants with weak continuity properties at singular edges (3D) and singular vertices (2D). We discuss implementation aspects that arise when coding the pressure space, and in particular, show that the pressure constraints can be enforced at an algebraic level.
\end{abstract}

divergence-free, low-order, Worsey-Farin, Powell-Sabin

\section{INTRODUCTION}

The first inf-sup stable finite element spaces that yield divergence-free approximations for incompressible fluid models on simplicial triangulations were given in the classical paper by Scott and Vogelius [15. Their lowest order pair, defined on a two-dimensional domain, used quartic Lagrage finite elements and piecewise cubic polynomials for the discrete velocity and pressure spaces, respectively; since then, there has been several efforts to find lower order inf-sup stable and conforming finite element spaces that produce divergence-free approximations [13, 14, 1, 18, 19, 20, 10, 11, 21.

If we confine ourselves to Lagrange elements for the velocity space, then a reduction of polynomial degree, while preserving stability, can be done on certain splits (i.e., refinements) of a simplicial mesh. For example, Zhang proved stability of the three-dimensional cubic-quadratic pair on Alfeld splits [18, and stability of the three-dimensional quadratic-linear pair on Worsey-Farin splits [21. On the other hand, Zhang suggests that piecewise linear Lagrange elements for the velocity are unstable on Worsey-Farin splits [21, p.244]. One of the main objectives of this paper is to show that indeed the linear case is stable if coupled with the correct pressure space.

The main goal of this paper is to construct inf-sup stable and divergence-free pairs on simplicial (split) meshes using a linear-constant velocity-pressure pair on Worsey-Farin splits (3D) and PowellSabin splits (2D). As far as we are aware, this is the first three-dimensional pair with these properties using linear Lagrange elements for the discrete velocity space. The main driver of our construction is an explicit characterization of the divergence operator acting on the Lagrange finite element space developed in 8, 9. Such characterizations naturally lead to the definitions of the discrete pressure space. In particular, the pressure space is a subspace of piecewise constants with weak continuity constraints on singular edges (Worsey-Farin) and singular vertices (Powell-Sabin).

Our results in two dimensions are similar to those in [19, where Zhang showed stability of a Stokes pair with linear Lagrange elements for the velocity space on Powell-Sabin splits. However, the pressure space was not characterized in [19] and as a result, a pressure basis is not explicitly constructed. Due to the implicit definition of the pressure space, one is forced to solve the resulting algebraic system by the iterated penalty method (IPM). In contrast, the explicit characterization of the pressure space we provide opens the door to a library of solvers based on the standard mixed formulation.

J. Guzmán was partially supported by NSF grant DMS-1913083,

M. Neilan and A. Zytoon was partially supported by NSF grant DMS-2011733, 
An advantage of the proposed schemes is their low computational cost and simple velocity spaces, which are supported in finite element software libraries. On the other hand, the discrete pressure spaces are subspaces of piecewise constants with weak continuity properties at singular edges and singular vertices; these spaces are nonstandard, and in particular are not readily available on computational software packages. Nonetheless, we show that these weak continuity properties can be enforced entirely at the algebraic level. One can simply form the algebraic saddle point problem using the full (unstable) linear-constant pair, and then perform elementary row and column operations to this system to enforce the weak continuity constraint. As a result, the proposed discretizations can be implemented on standard finite element software packages (e.g., FEniCS).

We note that degrees of freedom and commuting projections were given in [8, 9]. In fact, an entire exact sequence of spaces were presented. However, stability (e.g., inf-sup stability) was not shown, which is something we carry out here.

Moreover, we provide numerical experiments to validate our theoretical results. In particular, we show that the computed solution is satisfying the standard error estimates in mixed formulation. Also, we provide a time comparison between the stiffness matrix modification method described above and the iterated penalty method.

The paper is organized as follows. In the next section we give notation that will be used in the rest of the paper. In Section 2 we provide the notation used throughout the paper and introduce the Stokes problem. In Section 3 prove the inf-sup stability of a low-order finite element pair on Powell-Sabin splits. In Section 4 we prove stability of the analogous three-dimensional pair on Worsey-Farin splits. In Section 5 we discuss implementation aspects on Powell-Sabin splits and in Section 6 we do the same for Worsey-Farin splits. Finally, in Section 7 we provide numerical experiments.

\section{Preliminaries}

In this section we develop basic notation that we use throughout the paper. We provide this in the following list:

- $\mathcal{T}_{h}$ is a shape-regular, simplicial triangulation of a contractible polytope $\Omega \subset \mathbb{R}^{d}(d=2,3)$.

- $h_{T}=\operatorname{diam}(T)$ for all $T \in \mathcal{T}_{h}$ and $h=\max _{T \in \mathcal{T}_{h}} h_{T}$.

- For an $n$-dimensional simplex $S(n \leq d)$ and $m \in\{0, \ldots n\}$, denote by $\Delta_{m}(S)$ the set of $m$-dimensional simplices of $S$.

- $\mathcal{P}_{r}(S)$ denotes the space of polynomials of degree $\leq r$ with domain $S$. Analogous spaces of vector-valued spaces are given in boldface, e.g., $\mathcal{P}_{r}(S)=\left[\mathcal{P}_{r}(S)\right]^{d}$.

- We define the following function spaces on $\Omega$ :

$$
\begin{aligned}
L^{2}(\Omega) & :=\left\{w: \Omega \mapsto \mathbb{R}:\|w\|_{L^{2}(\Omega)}:=\left(\int_{\Omega}|w|^{2} d x\right)^{1 / 2}<\infty\right\}, \\
H^{m}(\Omega) & :=\left\{w: \Omega \mapsto \mathbb{R}:\|w\|_{H^{m}(\Omega)}:=\left(\sum_{|\beta| \leq m}\left\|D^{\beta} w\right\|_{L^{2}(\Omega)}^{2}\right)^{1 / 2}<\infty\right\},
\end{aligned}
$$

and the spaces with boundary conditions:

$$
\begin{aligned}
L_{0}^{2}(\Omega) & :=\left\{w \in L^{2}(\Omega): \int_{\Omega} w d x=0\right\} \\
H_{0}^{m}(\Omega) & :=\left\{w \in H^{m}(\Omega):\left.D^{\beta} w\right|_{\partial \Omega}=0, \forall \beta:|\beta| \leq m-1\right\} .
\end{aligned}
$$

Analogous vector-valued spaces are denoted in boldface, e.g., $\boldsymbol{H}^{1}(\Omega)=\left[H^{1}(\Omega)\right]^{d}$.

- For a simplicial triangulation $Q_{h}$ of $\Omega$ we define the spaces of piecewise polynomials

$$
\begin{array}{ll}
\mathcal{P}_{k}\left(\mathcal{Q}_{h}\right)=\prod_{K \in \mathcal{Q}_{h}} \mathcal{P}_{k}(K), & \mathcal{P}_{k}\left(\mathcal{Q}_{h}\right)=\prod_{K \in \mathcal{Q}_{h}} \mathcal{P}_{k}(K), \\
\stackrel{\circ}{\mathcal{P}}_{k}\left(\mathcal{Q}_{h}\right)=\mathcal{P}_{k}\left(\mathcal{Q}_{h}\right) \cap L_{0}^{2}(\Omega), & \stackrel{\mathcal{P}}{k}_{k}^{c}\left(\mathcal{Q}_{h}\right)=\mathcal{P}_{k}\left(\mathcal{Q}_{h}\right) \cap \boldsymbol{H}_{0}^{1}(\Omega) .
\end{array}
$$


Thus $\stackrel{\circ}{\mathcal{P}}_{k}\left(Q_{h}\right)$ consists of piecewise polynomials of degree $\leq k$ with respect to the triangulation $Q_{h}$ with mean zero, and $\stackrel{\mathcal{P}}{k}_{k}^{c}\left(Q_{h}\right)$ is the space of continuous, piecewise polynomials of degree $\leq k$ with vanishing trace (i.e., the $k$ th degree vector-valued Lagrange finite element space).

- The constant $C$ denotes a generic positive constant, independent of the mesh parameter $h$.

2.1. The Stokes problem. The Stokes equations defined on a polygonal domain $\Omega \subset \mathbb{R}^{d}$ with Lipschitz continuous boundary $\partial \Omega$ is given by the system of equations

$$
\begin{array}{rlrl}
-\nu \Delta \boldsymbol{u}+\nabla p & =\boldsymbol{f} & & \text { in } \Omega, \\
\operatorname{div} \boldsymbol{u}=0 & & \text { in } \Omega, \\
\boldsymbol{u} & =0 & & \text { on } \partial \Omega,
\end{array}
$$

where the velocity $\boldsymbol{u}=\left(u_{1}, \ldots, u_{d}\right)^{\boldsymbol{\top}}$ and pressure $p$ are functions of $x=\left(x_{1}, \ldots, x_{d}\right)^{\boldsymbol{\top}}$, and $\nabla, \Delta$ denote the gradient operator and vector Laplacian operator with respect to $x$ respectively. In (2.1a), $\nu$ is the viscosity.

The weak formulation for (2.1) reads: Find $(\boldsymbol{u}, p) \in \boldsymbol{H}_{0}^{1}(\Omega) \times L_{0}^{2}(\Omega)$ such that $\forall(\boldsymbol{v}, q) \in \boldsymbol{H}_{0}^{1}(\Omega) \times$ $L_{0}^{2}(\Omega)$ we have

$$
\begin{aligned}
\nu \int_{\Omega} \nabla \boldsymbol{u}: \nabla \boldsymbol{v}-\int_{\Omega}(\operatorname{div} \boldsymbol{v}) p & =\int_{\Omega} \boldsymbol{f} \cdot \boldsymbol{v}, \\
\int_{\Omega}(\operatorname{div} \boldsymbol{u}) q & =0 .
\end{aligned}
$$

It is well known that the problem (2.2) has a unique solution [7].

Let $\stackrel{\circ}{V}_{h} \times \stackrel{\circ}{Y}_{h} \subset \boldsymbol{H}_{0}^{1}(\Omega) \times L_{0}^{2}(\Omega)$ be a conforming and finite dimensional pair. Then the finite element method for (2.1), based on the standard velocity-pressure formulation, seeks $\left(\boldsymbol{u}_{h}, p_{h}\right) \in \stackrel{\circ}{\boldsymbol{V}}_{h} \times \dot{Y}_{h}$ such that $\forall(\boldsymbol{v}, q) \in{\stackrel{\circ}{\boldsymbol{V}_{h}}} \times \stackrel{\circ}{Y}_{h}$ we have

$$
\begin{aligned}
\nu \int_{\Omega} \nabla \boldsymbol{u}_{h}: \nabla \boldsymbol{v}- & \int_{\Omega}(\operatorname{div} \boldsymbol{v}) p_{h}=\int_{\Omega} \boldsymbol{f} \cdot \boldsymbol{v}, \\
\int_{\Omega}\left(\operatorname{div} \boldsymbol{u}_{h}\right) q & =0 .
\end{aligned}
$$

The discrete problem (2.3) is well-posed if and only if the pair satisfies the inf-sup condition

$$
\sup _{0 \neq \boldsymbol{v} \in \dot{\boldsymbol{V}}_{h}} \frac{\int_{\Omega}(\operatorname{div} \boldsymbol{v}) q}{\|\nabla \boldsymbol{v}\|_{L^{2}(\Omega)}} \geq \beta\|q\|_{L^{2}(\Omega)},
$$

for some $\beta>0$.

\section{Inf-Sup stability on Powell-Sabin Triangulations}

Let $\mathcal{T}_{h}$ be a simplicial, shape-regular triangulation of $\Omega \subset \mathbb{R}^{2}$. The Powell-Sabin refinement of $\mathcal{T}_{h}$, denoted by $\mathcal{T}_{h}^{\mathrm{PS}}$ is obtained by the following procedure. First connect the incenter of each triangle $T \in \mathcal{T}_{h}$ with its three vertices. Next, the incenters of each adjacent pair of triangles are connected with an edge. If $T$ has a boundary edge, then the midpoint of the boundary edge is connected to the incenter of $T$. Thus, this procedure splits each triangle in $\mathcal{T}_{h}$ into six subtriangles; cf. Figure 1.

One feature of the Powell-Sabin triangulation is the presence of singular vertices.

Definition 3.1. We say that a vertex in a simplicial triangulation is singular if the edges meeting at the vertex fall on exactly two straight lines.

Let $\mathcal{S}_{h}^{I}$ and $\mathcal{S}_{h}^{B}$ denote the sets of interior and boundary singular vertices in $\mathcal{T}_{h}^{\mathrm{PS}}$, respectively, and set $\mathcal{S}_{h}=\mathcal{S}_{h}^{I} \cup \mathcal{S}_{h}^{B}$. Note that the cardinalities of the sets $\mathcal{S}_{h}^{I}$ and $\mathcal{S}_{h}^{B}$ correspond to the number of interior and boundary edges in $\mathcal{T}_{h}$, respectively. For $z \in \mathcal{S}_{h}^{I}$, we denote by $\mathcal{T}_{z} \subset \mathcal{T}_{h}^{\text {PS }}$ the set of four triangles that have $z$ as a vertex. We write $\mathcal{T}_{z}=\left\{K_{z}^{(1)}, K_{z}^{(2)}, K_{z}^{(3)}, K_{z}^{(4)}\right\}$ with $K_{z}^{(j)} \in \mathcal{T}_{h}^{\text {PS }}$, labeled 

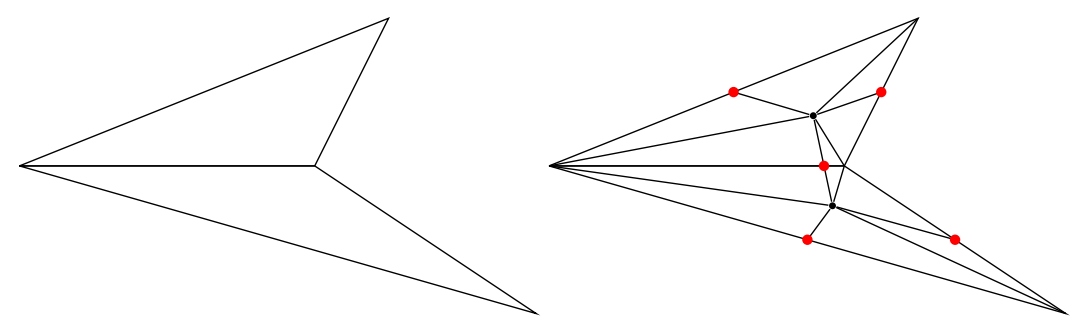

Figure 1. Two triangles in the mesh $\mathcal{T}_{h}$ (left) and their Powell-Sabin refinement (right). The singular vertices in the Powell-Sabin refinement are depicted in red.

such that $K_{z}^{(j)}$ and $K_{z}^{(j+1)}$ have a common edge. Likewise, for $z \in \mathcal{S}_{h}^{B}$, we set $\mathcal{T}_{z}=\left\{K_{z}^{(1)}, K_{z}^{(2)}\right\} \subset \mathcal{T}_{h}^{\mathrm{PS}}$ to denote the two triangles that have $z$ as a vertex. We set $n_{z}=\left|\mathcal{T}_{z}\right|$, i.e., $n_{z}=4$ if $z \in \mathcal{S}_{h}^{I}$ and $n_{z}=2$ if $z \in \mathcal{S}_{h}^{B}$.

3.1. Finite Element Spaces on Powell-Sabin Triangulations. It is well known that, on singular vertices, the divergence operator acting on the Lagrange finite element space has "weak continuity properties"; the precise meaning of this statement is provided in the following lemma. Its proof can be found in, e.g., [15, 12.

Lemma 3.2. For $z \in \mathcal{S}_{h}$, and piecewise smooth function $q$, define

$$
\theta_{z}(q):= \begin{cases}\left.q\right|_{K_{z}^{(1)}}(z)-\left.q\right|_{K_{z}^{(2)}}(z)+\left.q\right|_{K_{z}^{(3)}}(z)-\left.q\right|_{K_{z}^{(4)}}(z) & z \in \mathcal{S}_{h}^{I}, \\ \left.q\right|_{K_{z}^{(1)}}(z)-\left.q\right|_{K_{z}^{(2)}} ^{(2)} & z \in \mathcal{S}_{h}^{B} .\end{cases}
$$

Then there holds $\theta_{z}(\operatorname{div} \boldsymbol{v})=0$ for all $\boldsymbol{v} \in \stackrel{\stackrel{\mathcal{P}}{c}}{k}^{c}\left(\mathcal{T}_{h}^{\mathrm{PS}}\right)$.

Based on Lemma 3.2, and on a discrete de Rham complex, divergence-free finite element pairs for the Stokes problem have been constructed and analyzed in [8, 19] on Powell-Sabin triangulations. Here, we focus on the lowest-order case, where the velocity space is the linear Lagrange space, and the pressure space is a subspace of piecewise constants with a weak continuity property at singular vertices. We first define the spaces without boundary conditions and then the ones with boundary conditions.

$$
\begin{aligned}
V_{h}^{\mathrm{PS}} & :=\mathcal{P}_{1}^{c}\left(\mathcal{T}_{h}^{\mathrm{PS}}\right), \\
Y_{h}^{\mathrm{PS}} & :=\left\{q \in \mathcal{P}_{0}\left(\mathcal{T}_{h}^{\mathrm{PS}}\right): \theta_{z}(q)=0 \forall z \in \mathcal{S}_{h}^{I}\right\} .
\end{aligned}
$$

We now define an intermediate pressure space

$$
\hat{Y}_{h}^{\mathrm{PS}}:=\left\{q \in \mathcal{P}_{0}\left(\mathcal{T}_{h}^{\mathrm{PS}}\right): \theta_{z}(q)=0 \forall z \in \mathcal{S}_{h}\right\} .
$$

The spaces with boundary conditions are

$$
\begin{aligned}
& {\stackrel{\circ}{\boldsymbol{V}_{h}}}^{\mathrm{PS}}:=\boldsymbol{V}_{h}^{\mathrm{PS}} \cap \boldsymbol{H}_{0}^{1}(\Omega), \\
& \stackrel{\circ}{Y}^{\mathrm{PS}}:=\hat{Y}_{h}^{\mathrm{PS}} \cap L_{0}^{2}(\Omega) .
\end{aligned}
$$

3.2. Stability of $\dot{\boldsymbol{V}}_{h}^{\mathrm{PS}} \times \stackrel{\circ}{Y}_{h}^{\mathrm{PS}}$. The stability of the pair $\stackrel{\circ}{h}_{h}^{\mathrm{PS}} \times \stackrel{\circ}{Y}_{h}^{\mathrm{PS}}$ is implicitly shown in [2]. Here we give more details.

Theorem 3.3. There holds $\operatorname{div} \stackrel{\circ}{V}_{h}^{\mathrm{PS}} \subseteq \stackrel{\circ}{Y}_{h}^{\mathrm{PS}}$. Moreover, the pair $\stackrel{\circ}{h}^{\mathrm{PS}} \times \stackrel{\circ}{Y}_{h}^{\mathrm{PS}}$ is an inf-sup stable pair for the Stokes problem, i.e.,

$$
\sup _{0 \neq \boldsymbol{v} \in \dot{\boldsymbol{V}}_{h}^{\mathrm{PS}}} \frac{\int_{\Omega}(\operatorname{div} \boldsymbol{v}) q}{\|\nabla \boldsymbol{v}\|_{L^{2}(\Omega)}} \geq \beta\|q\|_{L^{2}(\Omega)} \quad \forall q \in \stackrel{\circ}{h}^{\mathrm{PS}},
$$

for some $\beta>0$ independent of the mesh parameter $h$. 
Proof. For fixed $q \in \stackrel{\circ}{Y}_{h}^{\mathrm{PS}}$, let $\boldsymbol{w} \in \boldsymbol{H}_{0}^{1}(\Omega)$ satisfy $\operatorname{div} \boldsymbol{w}=q$ and $\|\boldsymbol{w}\|_{H^{1}(\Omega)} \leq C\|q\|_{L^{2}(\Omega)}$. 2, Theorem 3.4] gives the existence of an operator $\boldsymbol{\Pi}_{h}$ such that $\boldsymbol{\Pi}_{h} \boldsymbol{w} \in \boldsymbol{V}_{h}^{\mathrm{PS}}, \operatorname{div} \boldsymbol{\Pi}_{h} \boldsymbol{w}=\operatorname{div} \boldsymbol{w}=q$, and

$$
\left\|\boldsymbol{w}-\Pi_{h} \boldsymbol{w}\right\|_{L^{2}(T)} \leq C h_{T}\left[\|\boldsymbol{w}\|_{H^{1}(\omega(T))}+\|\operatorname{div} \boldsymbol{w}\|_{L^{2}(T)}\right] \quad \forall T \in \mathcal{T}_{h},
$$

where $\omega(T)=\cup_{T^{\prime} \in \mathcal{T}_{h} \bar{T}^{\prime} \cap \bar{T} \neq \emptyset} T^{\prime}$. Standard arguments then show $\left\|\nabla \boldsymbol{\Pi}_{h} \boldsymbol{w}\right\|_{L^{2}(\Omega)} \leq C\left(\|\boldsymbol{w}\|_{H^{1}(\Omega)}+\right.$ $\left.\|\operatorname{div} \boldsymbol{w}\|_{L^{2}(\Omega)}\right) \leq C\|q\|_{L^{2}(\Omega)}$. Setting $\boldsymbol{v}=\boldsymbol{\Pi}_{h} \boldsymbol{w}$, we have

$$
\int_{\Omega}(\operatorname{div} \boldsymbol{v}) q d x=\int_{\Omega}\left(\operatorname{div} \boldsymbol{\Pi}_{h} \boldsymbol{w}\right) q d x=\|q\|_{L^{2}(\Omega)}^{2} \geq C^{-1}\|q\|_{L^{2}(\Omega)}\|\nabla \boldsymbol{v}\|_{L^{2}(\Omega)} .
$$

This yields the inf-sup stability result with $\beta=C^{-1}$.

\section{INF-SUP STABILITY ON WORSEY-FARIN SPLITS}

Let $\mathcal{T}_{h}$ be a simplicial triangulation of a polyhedral domain $\Omega \subset \mathbb{R}^{3}$. The Worsey-Farin triangulation $\mathcal{T}_{h}^{\mathrm{WF}}$ is obtained by splitting each tetrahedron into twelve sub-tetrahedra by the following procedure. Similar to the Powell-Sabin case, for each $T \in \mathcal{T}_{h}$, we connect the incenter of $T$ to its vertices. Next, the incenters of neighboring pairs of tetrahedra are connected with an edge. This creates a face split point (a vertex) on each face of $T$. If $T$ has a boundary face, then we connect the incenter of $T$ to the barycenter of the face by a line. Finally, the face split points are connected to the vertices of the face. For each $T \in \mathcal{T}_{h}$, we denote by $T^{\mathrm{WF}}$ the triangulation resulting from local Worsey-Farin refinement of $T$, i.e.,

$$
T^{\mathrm{WF}}=\left\{K \in \mathcal{T}_{h}^{\mathrm{WF}}: K \subset \bar{T}\right\} .
$$

Definition 4.1. An edge in a 3D simplicial triangulation is called singular if the faces meeting at the edge fall on exactly two planes.

By construction, the Worsey-Farin triangulation contains many singular edges; for each face in the unrefined triangulation $\mathcal{T}_{h}$, there are three associated singular edges in $\mathcal{T}_{h}^{\mathrm{WF}}$.

Let $\mathcal{E}_{h}^{\mathcal{S}}$ denote the set of singular edges in $\mathcal{T}_{h}^{\mathrm{WF}}$, and let $\mathcal{E}_{h}^{\mathcal{S}, I}$ and $\mathcal{E}_{h}^{\mathcal{S}, B}$ denote the sets of interior and boundary singular edges, respectively. For each $e \in \mathcal{E}_{h}^{\mathcal{S}}$, let $\mathcal{T}_{e}=\left\{K_{e}^{(1)}, \ldots, K_{e}^{\left(n_{e}\right)}\right\}$ denote the set of tetrahedra that have $e$ as an edge. Here, $n_{e}=4$ if $e$ is an interior edge, and $n_{e}=2$ if $e$ is a boundary edge. We assume the tetrahedra are labeled such that $K_{e}^{(j)}$ and $K_{e}^{(j+1)}$ share a common face.

4.1. Finite Element Spaces on Worsey-Farin Triangulations. Similar to the two-dimensional case, the divergence operator acting on the Lagrange finite element space has weak continuity properties on singular edges (cf. 9]).

Lemma 4.2. For $e \in \mathcal{E}_{h}^{S}$, and a piecewise smooth function $q$, define

$$
\theta_{e}(q)= \begin{cases}\left.q_{e}^{(1)}\right|_{e}-\left.q_{e}^{(2)}\right|_{e}+\left.q_{e}^{(3)}\right|_{e}-\left.q_{e}^{(4)}\right|_{e} & e \in \mathcal{E}_{h}^{\mathcal{S}, I}, \\ \left.q_{e}^{(1)}\right|_{e}-\left.q_{e}^{(2)}\right|_{e} & e \in \mathcal{E}_{h}^{\mathcal{S}, B},\end{cases}
$$

where $q_{e}^{(j)}=\left.q\right|_{K_{e}^{(j)}}$. Then there holds $\theta_{e}(\operatorname{div} \boldsymbol{v})=0$ for all $\boldsymbol{v} \in \stackrel{\mathcal{P}}{c}_{k}^{c}\left(\mathcal{T}_{h}^{\mathrm{WF}}\right)$.

Analogous to the Powell-Sabin case, we define the finite element spaces to discretize the Stokes problem on Worsey-Farin splits. We first define the spaces without boundary conditions

$$
\begin{aligned}
& \boldsymbol{V}_{h}^{\mathrm{WF}}=\mathcal{P}_{1}^{c}\left(\mathcal{T}_{h}^{\mathrm{WF}}\right), \\
& Y_{h}^{\mathrm{WF}}=\left\{q \in \stackrel{\mathcal{P}}{0}_{0}\left(\mathcal{T}_{h}^{\mathrm{WF}}\right): \theta_{e}(q)=0 \forall e \in \mathcal{E}_{h}^{\mathcal{S}, I}\right\} .
\end{aligned}
$$

Then, we define an intermediate pressure space

$$
\hat{Y}_{h}^{\mathrm{WF}}=\left\{q \in \stackrel{\circ}{\mathcal{P}}_{0}\left(\mathcal{T}_{h}^{\mathrm{WF}}\right): \theta_{e}(q)=0 \forall e \in \mathcal{E}_{h}^{\mathcal{S}}\right\} .
$$


We now define the spaces with boundary conditions

$$
\begin{aligned}
& \stackrel{\circ}{h}^{\mathrm{WF}}=\boldsymbol{V}_{h}^{\mathrm{WF}} \cap \boldsymbol{H}_{0}^{1}(\Omega), \\
& \stackrel{\circ}{Y}^{\mathrm{WF}}=\hat{Y}_{h}^{\mathrm{WF}} \cap L_{0}^{2}(\Omega) .
\end{aligned}
$$

4.2. Stability of $\dot{\boldsymbol{V}}_{h}^{\mathrm{WF}} \times \stackrel{\circ}{Y}_{h}^{\mathrm{WF}}$. In this section, we show the pair $\stackrel{\circ}{\boldsymbol{V}}_{h}^{\mathrm{WF}} \times \dot{\circ}_{h}^{\mathrm{WF}}$ is inf-sup stable. First we introduce some notation.

Let $T \in \mathcal{T}_{h}$, and let $T^{\mathrm{A}}$ denote the local triangulation of $T$ consisting of four tetrahedra, obtained by connecting the vertices of $T$ with its incenter, i.e., $T^{\mathrm{A}}$ denotes the Alfeld split of $T$. For a face $F \subset T$, denote by $F^{\mathrm{CT}}$ the set of three triangles formed from $F$ by the Worsey-Farin refinement, i.e., $F^{\mathrm{CT}}$ is the Clough-Tocher refinement of $F$. We denote by $\Delta_{1}^{I}\left(F^{\mathrm{CT}}\right)$ the set of three interior edges in $F^{\mathrm{CT}}$, and let $e_{F} \in \Delta_{1}^{I}\left(F^{\mathrm{CT}}\right)$ denote an arbitrary, fixed interior edge of $F^{\mathrm{CT}}$.

Definition 4.3. Consider the triangulation $F^{C T}$ of a face $F \in \Delta_{2}(T)$, and let the three triangles of $F^{\mathrm{CT}}$ be labeled $Q_{1}, Q_{2}, Q_{3}$. Let $e=\partial Q_{1} \cap \partial Q_{2}$ be an internal edge, let $\boldsymbol{t}$ be the unit vector tangent to $e$ pointing away from the split point $m_{F}$, and let $s$ be the unit vector orthogonal to $t$ such that $\boldsymbol{s} \times \boldsymbol{n}_{F}=\boldsymbol{t}$. Then the jump of a piecewise smooth function $p$ across $e$ is defined as

$$
\llbracket p \rrbracket_{e}=\left(\left.p\right|_{Q_{1}}-\left.p\right|_{Q_{2}}\right) s .
$$

We now state the degrees of freedom for the spaces $\stackrel{\circ}{V}_{h}^{\mathrm{WF}}$ and $\stackrel{\circ}{Y}_{h}^{\mathrm{WF}}$. The proofs of the following two lemmas are given in [9, Lemmas 5.11-5.12].

Lemma 4.4. A function $\boldsymbol{v} \in \boldsymbol{V}_{h}^{\mathrm{WF}}$ is uniquely determined by the values

$$
\begin{array}{ll}
\boldsymbol{v}(a) & \forall a \in \Delta_{0}(T), \\
\int_{F}\left(\boldsymbol{v} \cdot \boldsymbol{n}_{F}\right) & \forall F \in \Delta_{2}(T), \\
\int_{e} \llbracket \operatorname{div} \boldsymbol{v} \rrbracket_{e} & \forall e \in \Delta_{1}^{I}\left(F^{\mathrm{CT}}\right) \backslash\left\{e_{F}\right\}, \forall F \in \Delta_{2}(T), \\
\int_{T}(\operatorname{div} \boldsymbol{v}) p & \forall p \in \dot{\mathcal{V}}_{0}(T):=\mathcal{P}_{0}\left(T^{\mathrm{A}}\right) \cap L_{0}^{2}(T) .
\end{array}
$$

for each $T \in \mathcal{T}_{h}$.

Lemma 4.5. A function $q \in Y_{h}^{\mathrm{WF}}$ is uniquely determined by

$$
\begin{array}{ll}
\int_{e} \llbracket q \rrbracket_{e} & \forall e \in \Delta_{1}^{I}\left(F^{\mathrm{CT}}\right) \backslash\left\{e_{F}\right\}, \forall F \in \Delta_{2}(T), \\
\int_{T} q p & \forall p \in \mathcal{P}_{0}\left(T^{\mathrm{A}}\right) .
\end{array}
$$

for all $T \in \mathcal{T}_{h}$.

If we restrict ourselves to $\hat{Y}_{h}^{\mathrm{WF}}$ then we only take interior faces $F$ in the first set of degrees of freedom.

Proposition 4.6. Let $\boldsymbol{v} \in \boldsymbol{V}_{h}^{\mathrm{WF}}$ and $T \in \mathcal{T}_{h}$. For $m=0,1$, there holds

$$
|\boldsymbol{v}|_{H^{m}(T)} \leq C h_{T}^{-1-2 m}\left(h_{T}^{4} \sum_{a \in \Delta_{0}(T)}|\boldsymbol{v}(a)|^{2}+\sum_{F \in \Delta_{2}(T)}\left|\int_{F} \boldsymbol{v} \cdot \boldsymbol{n}_{F}\right|^{2}+h_{T}^{3}\|\operatorname{div} \boldsymbol{v}\|_{L^{2}(T)}^{2}\right) .
$$

Proof. Let $\hat{T}$ be the reference tetrahedron, and let $F_{T}: \hat{T} \rightarrow T$ be an affine bijection with $F_{T}(\hat{x})=$ $A_{T} \hat{x}+b_{T}$ with $A_{T} \in \mathbb{R}^{3 \times 3}$ and $b_{T} \in \mathbb{R}^{3}$. We define $\hat{\boldsymbol{v}}: \hat{T} \rightarrow \mathbb{R}^{3}$ via the Piola transform

$$
\boldsymbol{v}(x)=\frac{A_{T} \hat{\boldsymbol{v}}(\hat{x})}{\operatorname{det}\left(A_{T}\right)}, \quad x=F_{T}(\hat{x}) .
$$


Let $\hat{T}^{\mathrm{WF}}$ be the split of $\hat{T}$ induced by $T^{\mathrm{WF}}$ and the mapping $F_{T}^{-1}$, i.e.,

$$
\hat{T}^{\mathrm{WF}}=\left\{F_{T}^{-1}(K): K \in T^{\mathrm{WF}}\right\} .
$$

Then $\hat{\boldsymbol{v}}$ is a continuous piecewise linear polynomial with respect to $\hat{T}^{\mathrm{WF}}$, and therefore by equivalence of norms, and Lemma 4.4 ,

$$
\begin{aligned}
|\hat{\boldsymbol{v}}|_{H^{m}(\hat{T})}^{2} \leq & C \sum_{\hat{a} \in \Delta_{0}(\hat{T})}|\hat{\boldsymbol{v}}(\hat{a})|^{2}+\sum_{\hat{F} \in \Delta_{2}(\hat{T})}\left|\int_{\hat{F}} \hat{\boldsymbol{v}} \cdot \hat{\boldsymbol{n}}_{\hat{F}}\right|^{2} \\
& \left.+\sum_{\hat{F} \in \Delta_{2}(\hat{T})} \sum_{\hat{e} \in \Delta_{1}^{I}\left(\hat{F}^{\mathrm{CT}}\right) \backslash\left\{\hat{e}_{\hat{F}}\right\}}\left|\int_{\hat{e}}[[\widehat{\operatorname{div}} \hat{\boldsymbol{v}}]]_{\hat{e}}\right|^{2}+\sup _{\substack{\hat{p} \in \dot{\mathcal{V}}_{0}(\hat{T}) \\
\|\hat{p}\|_{L^{2}(\hat{T})}=1}}\left|\int_{\hat{T}}(\widehat{\operatorname{div}} \hat{\boldsymbol{v}}) \hat{p}\right|^{2}\right) .
\end{aligned}
$$

By well-known properties of the Piola transform, we have

$$
\operatorname{div} \boldsymbol{v}(x)=\frac{1}{\operatorname{det}\left(A_{T}\right)} \widehat{\operatorname{div}} \hat{\boldsymbol{v}}(\hat{x}), \quad \int_{F} \boldsymbol{v} \cdot \boldsymbol{n}_{F}=\int_{\hat{F}} \hat{\boldsymbol{v}} \cdot \hat{\boldsymbol{n}}_{\hat{F}} .
$$

Thus, we have

$$
\begin{aligned}
|\hat{\boldsymbol{v}}|_{H^{m}(\hat{T})}^{2} \leq & C\left(\sum_{a \in \Delta_{0}(T)}\left|\operatorname{det}\left(A_{T}\right) A_{T}^{-1} \boldsymbol{v}(a)\right|^{2}+\sum_{F \in \Delta_{2}(T)}\left|\int_{F} \boldsymbol{v} \cdot \boldsymbol{n}_{F}\right|^{2}\right. \\
& \left.+\left|\operatorname{det}\left(A_{T}\right)\right|^{2} \sum_{F \in \Delta_{2}(T)} \sum_{e \in \Delta_{1}(F) \backslash\left\{e_{F}\right\}}\left|\frac{|\hat{e}|}{|e|} \int_{e} \llbracket \operatorname{div} \boldsymbol{v} \rrbracket_{e}\right|^{2}+\sup _{\substack{\hat{p} \in \hat{V}_{0}(\hat{T}) \\
\|\hat{p}\|_{L^{2}(\hat{T})}=1}}\left|\int_{\hat{T}}(\widehat{\operatorname{div}} \hat{\boldsymbol{v}}) \hat{p}\right|^{2}\right) .
\end{aligned}
$$

Next, for $\hat{p} \in \dot{\mathcal{V}}_{0}(\hat{T})$ with $\|\hat{p}\|_{L^{2}(\hat{T})}=1$, let $p: T \rightarrow \mathbb{R}$ be given by $p(x)=\hat{p}(\hat{x})$. Then $p \in \dot{\mathcal{V}}_{0}(T)$, $\|p\|_{L^{2}(T)}=\sqrt{6|T|}$, and

We conclude

$$
\int_{\hat{T}}(\widehat{\operatorname{div}} \hat{\boldsymbol{v}}) \hat{p}=\int_{T}(\operatorname{div} \boldsymbol{v}) p
$$

$$
\sup _{\substack{\hat{p} \in \dot{\mathcal{V}}_{0}(\hat{T}) \\\|\hat{p}\|_{L^{2}(\hat{T})}=1}}\left|\int_{\hat{T}}(\widehat{\operatorname{div}} \hat{\boldsymbol{v}}) \hat{p}\right|^{2} \leq \sup _{\substack{p \in \dot{\mathcal{V}}_{0}(T) \\\|p\|_{L^{2}(T)}=\sqrt{6|T|}}}\left|\int_{T}(\operatorname{div} \boldsymbol{v}) p\right|^{2} \leq C h_{T}^{3}\|\operatorname{div} \boldsymbol{v}\|_{L^{2}(T)}^{2} .
$$

Finally, we use $\left\|A_{T}^{-1}\right\| \leq C h_{T}^{-1}$ and $\left|\operatorname{det}\left(A_{T}\right)\right|=6|T| \leq C h_{T}^{3}$ to get

$$
\begin{aligned}
|\hat{\boldsymbol{v}}|_{H^{m}(\hat{T})}^{2} \leq & C\left(h_{T}^{4} \sum_{a \in \Delta_{0}(T)}|\boldsymbol{v}(a)|^{2}+\sum_{F \in \Delta_{2}(T)}\left|\int_{F} \boldsymbol{v} \cdot \boldsymbol{n}_{F}\right|^{2}\right. \\
& \left.+h_{T}^{4} \sum_{F \in \Delta_{2}(T)} \sum_{e \in \Delta_{1}^{I}\left(F^{\mathrm{CT}}\right) \backslash\left\{e_{F}\right\}}\left|\int_{e} \llbracket \operatorname{div} \boldsymbol{v} \rrbracket_{e}\right|^{2}+h_{T}^{3}\|\operatorname{div} \boldsymbol{v}\|_{L^{2}(T)}^{2}\right),
\end{aligned}
$$

and therefore

$$
\begin{aligned}
|\boldsymbol{v}|_{H^{m}(\hat{T})}^{2} \leq C h_{T}^{-1-2 m}|\hat{\boldsymbol{v}}|_{H^{m}(\hat{T})}^{2} \leq & C h_{T}^{-1-2 m}\left(h_{T}^{4} \sum_{a \in \Delta_{0}(T)}|\boldsymbol{v}(a)|^{2}+\sum_{F \in \Delta_{2}(T)}\left|\int_{F} \boldsymbol{v} \cdot \boldsymbol{n}_{F}\right|^{2}\right. \\
& \left.\quad+h_{T}^{4} \sum_{F \in \Delta_{2}(T)} \sum_{e \in \Delta_{1}(F) \backslash\left\{e_{F}\right\}}\left|\int_{e} \llbracket \operatorname{div} \boldsymbol{v} \rrbracket_{e}\right|^{2}+h_{T}^{3}\|\operatorname{div} \boldsymbol{v}\|_{L^{2}(T)}^{2}\right) \\
\leq & C h_{T}^{-1-2 m}\left(h_{T}^{4} \sum_{a \in \Delta_{0}(T)}|\boldsymbol{v}(a)|^{2}+\sum_{F \in \Delta_{2}(T)}\left|\int_{F} \boldsymbol{v} \cdot \boldsymbol{n}_{F}\right|^{2}+h_{T}^{3}\|\operatorname{div} \boldsymbol{v}\|_{L^{2}(T)}^{2}\right),
\end{aligned}
$$

where the last inequality comes from standard trace and inverse inequalities.

Theorem 4.7. The pair $\stackrel{\circ}{h}^{\mathrm{WF}} \times \dot{Y}_{h}^{\mathrm{WF}}$ is inf-sup stable. 
Proof. Fix a $q \in \stackrel{\circ}{Y}_{h}^{\mathrm{WF}}$, and let $\boldsymbol{w} \in \boldsymbol{H}_{0}^{1}(\Omega)$ satisfy $\operatorname{div} \boldsymbol{w}=q$ and $\|\nabla \boldsymbol{w}\|_{L^{2}(\Omega)} \leq C\left\|_{q}\right\|_{L^{2}(\Omega)}$. Let $\boldsymbol{w}_{h} \in \stackrel{\circ}{\mathcal{P}}_{1}\left(\mathcal{T}_{h}\right)$ be the Scott-Zhang interpolant of $\boldsymbol{w}$ with respect to $\mathcal{T}_{h}$. Define $\boldsymbol{v} \in \stackrel{\circ}{\mathcal{P}}_{1}\left(\mathcal{T}_{h}^{\mathrm{WF}}\right)$ such that

$$
\begin{array}{ll}
\boldsymbol{v}(a)=\boldsymbol{w}_{h}(a) & \forall a \in \Delta_{0}(T), \\
\int_{F}\left(\boldsymbol{v} \cdot \boldsymbol{n}_{F}\right)=\int_{F}\left(\boldsymbol{w} \cdot \boldsymbol{n}_{F}\right) & \forall F \in \Delta_{2}(T), \\
\int_{e} \llbracket \operatorname{div} \boldsymbol{v} \rrbracket_{e}=\int_{e} \llbracket q \rrbracket_{e} & \forall e \in \Delta_{1}^{I}\left(F^{\mathrm{CT}}\right) \backslash\left\{e_{F}\right\}, \forall F \in \Delta_{2}(T), \\
\int_{T}(\operatorname{div} \boldsymbol{v}) p=\int_{T} q p & \forall p \in \dot{\mathcal{V}}_{0}(T) .
\end{array}
$$

Noting $(\operatorname{div} \boldsymbol{v}-q) \in \boldsymbol{Y}_{h}^{\mathrm{WF}}$, and

$$
\begin{array}{ll}
\int_{e} \llbracket \operatorname{div} \boldsymbol{v}-q \rrbracket_{e}=0 & \forall e \in \Delta_{1}^{I}\left(F^{\mathrm{CT}}\right) \backslash\left\{e_{F}\right\}, \forall F \in \Delta_{2}(T), \\
\int_{T}(\operatorname{div} \boldsymbol{v}-q) p & \forall p \in \mathcal{P}_{0}\left(T^{\mathrm{A}}\right)
\end{array}
$$

for all $T \in \mathcal{T}_{h}$ (by the divergence theorem), we conclude $\operatorname{div} \boldsymbol{v}=q$ by Lemma 4.5 ,

We apply Proposition 4.6 to $\left(\boldsymbol{v}-\boldsymbol{w}_{h}\right)$ with $m=1$ :

$$
\begin{aligned}
\left\|\nabla\left(\boldsymbol{v}-\boldsymbol{w}_{h}\right)\right\|_{L^{2}(T)}^{2} \leq & C h_{T}^{-3}\left(h_{T}^{4} \sum_{a \in \Delta_{0}(T)}\left|\left(\boldsymbol{v}-\boldsymbol{w}_{h}\right)(a)\right|^{2}+\sum_{F \in \Delta_{2}(T)}\left|\int_{F}\left(\boldsymbol{v}-\boldsymbol{w}_{h}\right) \cdot \boldsymbol{n}_{F}\right|^{2}\right. \\
& \left.\quad+h_{T}^{3}\left\|\operatorname{div}\left(\boldsymbol{v}-\boldsymbol{w}_{h}\right)\right\|_{L^{2}(T)}^{2}\right) \\
= & C h_{T}^{-3}\left(\sum_{F \in \Delta_{2}(T)}\left|\int_{F}\left(\boldsymbol{w}-\boldsymbol{w}_{h}\right) \cdot \boldsymbol{n}_{F}\right|^{2}+h_{T}^{3}\left\|q-\operatorname{div} \boldsymbol{w}_{h}\right\|_{L^{2}(T)}^{2}\right) \\
\leq & C h_{T}^{-3}\left(h_{T}^{2} \sum_{F \in \Delta_{2}(T)}\left\|\boldsymbol{w}-\boldsymbol{w}_{h}\right\|_{L^{2}(F)}^{2}+h_{T}^{3}\left\|q-\operatorname{div} \boldsymbol{w}_{h}\right\|_{L^{2}(T)}^{2}\right) \\
\leq & C\left(\|q\|_{L^{2}(T)}^{2}+h_{T}^{-2}\left\|\boldsymbol{w}-\boldsymbol{w}_{h}\right\|_{L^{2}(T)}^{2}+\left\|\nabla\left(\boldsymbol{w}-\boldsymbol{w}_{h}\right)\right\|_{L^{2}(T)}^{2}+\left\|\nabla \boldsymbol{w}_{h}\right\|_{L^{2}(T)}^{2}\right) .
\end{aligned}
$$

We then sum over $T \in \mathcal{T}_{h}$ and apply stability and approximation properties of the Scott-Zhang interpolant to conclude $\|\nabla \boldsymbol{v}\|_{L^{2}(\Omega)} \leq C\|q\|_{L^{2}(\Omega)}$.

\section{Implementation Aspects for Powell-Sabin splits}

The only tricky part to implement these finite elements is the pressure spaces since they have non-standard constraints in their definitions. In this section and the subsequent one, we give details to form the algebraic system for the Stokes problem.

5.1. A basis for $\hat{Y}_{h}^{\mathrm{PS}}$ and the construction of the algebraic system. Recall that the space $\hat{Y}_{h}^{\mathrm{PS}}$ consists of piecewise constant functions satisfying a weak continuity property at vertices. Clearly, this is a non-standard space, and in particular the space is not explicitly found in current finite element software packages. Nonetheless, in this section, we identify a basis of the space $\stackrel{\circ}{h}_{h}^{\mathrm{PS}}$, and as a byproduct show that the weak continuity property $\theta_{z}(q)=0$ can be imposed purely at the algebraic level.

As a first step, we note that, by definition of the Powell-Sabin triangulation,

$$
\mathcal{T}_{h}^{\mathrm{PS}}=\left\{K_{z}^{(j)}: K_{z}^{(j)} \in \mathcal{T}_{z}, z \in \mathcal{S}_{h}\right\}
$$




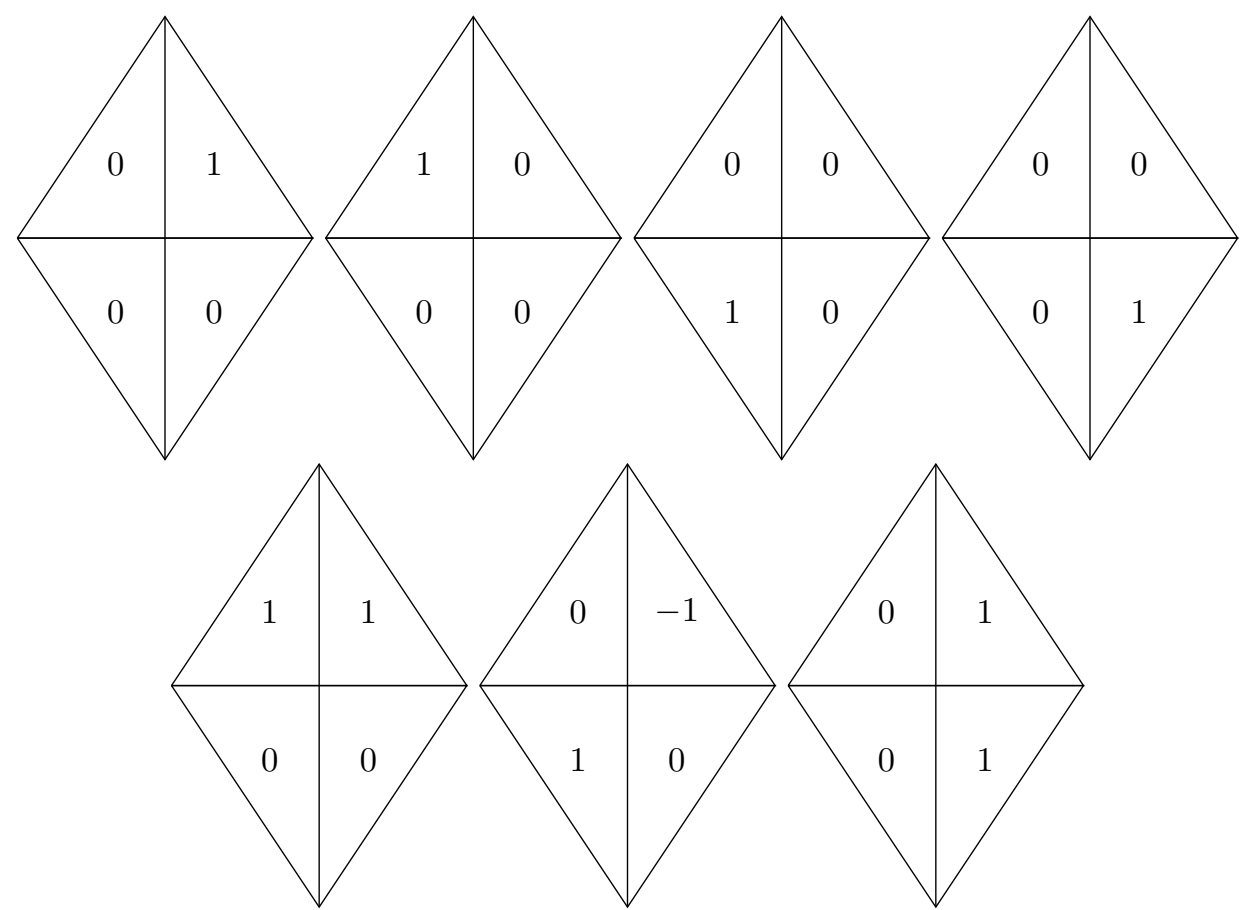

FiguRE 2. Local mesh $\mathcal{T}_{z}$ with $z \in \mathcal{S}_{h}^{I}$. Top row: Values of canonical basis functions of piecewise constants $\left\{\varphi_{z}^{(j)}\right\}_{j=1}^{n_{z}}$. Bottom row: Values of basis functions of piecewise constants with weak continuity constraint $\left\{\psi_{z}^{(j)}\right\}_{j=2}^{n_{z}}$.

With this (implicit) labeling of the triangles in $\mathcal{T}_{h}^{\mathrm{PS}}$, we can write the canonical basis of $\mathcal{P}_{0}\left(\mathcal{T}_{h}^{\mathrm{PS}}\right)$ as the set $\left\{\varphi_{z}^{(j)}\right\} \subset \mathcal{P}_{0}\left(\mathcal{T}_{h}^{\mathrm{PS}}\right)$ with

$$
\left.\varphi_{z}^{(j)}\right|_{K_{v}^{(i)}}=\delta_{v, z} \delta_{i, j} \quad \forall z, v \in \mathcal{S}_{h}, i=1, \ldots, n_{v}, j=1, \ldots, n_{z} .
$$

The next proposition shows that a basis of $\stackrel{\circ}{h}^{\mathrm{PS}}$ is easily extracted from the basis of $\mathcal{P}_{0}\left(\mathcal{T}_{h}^{\mathrm{PS}}\right.$ ) (see Figure 2).

Proposition 5.1. For each $z \in \mathcal{S}_{h}$ and $j \in\left\{2, \ldots, n_{z}\right\}$, define

$$
\psi_{z}^{(j)}=\varphi_{z}^{(j)}+(-1)^{j} \varphi_{z}^{(1)}
$$

Then $\left\{\psi_{z}^{(j)}: z \in \mathcal{S}_{h}, j=2, \ldots, n_{z}\right\}$ forms a basis of $\hat{Y}_{h}^{\mathrm{PS}}$.

Proof. Note that the number of functions $\left\{\psi_{z}^{(j)}\right\}$ given is $\sum_{z \in \mathcal{S}_{h}}\left(n_{z}-1\right)$, and

$$
\operatorname{dim} Y_{h}^{P S}=\operatorname{dim} \mathcal{P}_{0}\left(\mathcal{T}_{h}^{\mathrm{PS}}\right)-\left|\mathcal{S}_{h}\right|=\sum_{z \in \mathcal{S}_{h}} n_{z}-\left|\mathcal{S}_{h}\right|=\sum_{z \in \mathcal{S}_{h}}\left(n_{z}-1\right) .
$$

Because $\psi_{z}^{(j)} \in \stackrel{\circ}{Y}_{h}^{\mathrm{PS}}$, and they are clearly linear independent, we conclude that $\left\{\psi_{z}^{(j)}\right\}$ form a basis of $\stackrel{\circ}{Y}_{h}^{\mathrm{PS}}$.

We now explain how Proposition 5.1 provides a simple way to construct the stiffness matrix for the Stokes problem using the $\stackrel{\circ}{h}^{\mathrm{PS}} \times \stackrel{\circ}{Y}_{h}^{\mathrm{PS}}$ pair. To explain the procedure, we require some notation.

Let $A$ be the matrix associated with the bilinear form

$$
(\boldsymbol{v}, \boldsymbol{w}) \rightarrow \int_{\Omega} \nu \nabla \boldsymbol{v}: \nabla \boldsymbol{w} d x \quad \text { over } \boldsymbol{v}, \boldsymbol{w} \in \stackrel{\circ}{V}_{h}^{\mathrm{PS}},
$$


and let $\tilde{B}$ is the matrix associated with the bilinear form

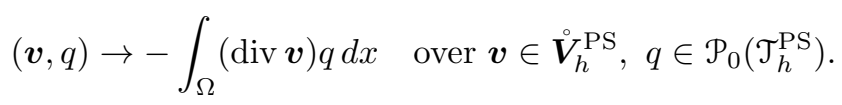

The stiffness matrix for the Stokes problem based on the (unstable) $\stackrel{\circ}{V}_{h}^{\mathrm{PS}} \times \mathcal{P}_{0}\left(\mathcal{T}_{h}^{\mathrm{PS}}\right)$ pair is given by

$$
\left(\begin{array}{cc}
A & \tilde{B} \\
\tilde{B}^{\top} & 0
\end{array}\right)
$$

We emphasize that this system can be easily constructed using standard finite element software packages.

Let $\left\{\boldsymbol{\phi}^{(i)}\right\}_{k=1}^{N}$ denote a basis of $\stackrel{\circ}{h}^{\mathrm{PS}}$ with $N=\operatorname{dim} \stackrel{\circ}{h}^{\mathrm{PS}}$ so that

$$
A_{i, j}=\nu \int_{\Omega} \nabla \phi^{(j)}: \nabla \phi^{(i)} d x .
$$

Let $M=\operatorname{dim} \mathcal{P}_{0}\left(\mathcal{T}_{h}^{\mathrm{PS}}\right)$, the number of triangles in $\mathcal{T}_{h}^{\text {PS }}$, and introduce the local-to-global label mapping $\sigma: \mathcal{S} \times\left\{1, \ldots, n_{z}\right\} \rightarrow\{1,2, \ldots, M\}$ such that

$$
\tilde{B}_{i, \sigma(z, j)}=-\int_{\Omega}\left(\operatorname{div} \phi^{(i)}\right) \varphi_{z}^{(j)} d x
$$

Then by Proposition 5.1 we have for $z \in \mathcal{S}_{h}$ and $j=2, \ldots, n_{z}$,

$$
\begin{aligned}
-\int_{\Omega}\left(\operatorname{div} \phi^{(i)}\right) \psi_{z}^{(j)} d x & =-\int_{\Omega}\left(\operatorname{div} \phi^{(i)}\right) \varphi_{z}^{(j)} d x-(-1)^{j} \int_{\Omega}\left(\operatorname{div} \phi^{(i)}\right) \varphi_{z}^{(1)} d x \\
& =\tilde{B}_{i, \sigma(z, j)}+(-1)^{j} \tilde{B}_{i, \sigma(z, 1) .}
\end{aligned}
$$

This identity leads to the following algorithm.

\section{Algorithm}

(1) Construct Powell-Sabin triangulation $\mathcal{T}_{h}^{\mathrm{PS}}$

(2) Construct $\tilde{B} \in \mathbb{R}^{N \times M}$ based on the $\stackrel{\circ}{h}^{\mathrm{PS}} \times \mathcal{P}_{0}\left(\mathcal{T}_{h}^{\mathrm{PS}}\right)$ pair.

(3) Set $B=\tilde{B}$.

(4) For each $z \in \mathcal{S}_{h}$ and for each $j \in\left\{2, \ldots, n_{z}\right\}$, do the elementary column operation

$$
B_{:, \sigma(z, j)}=B_{:, \sigma(z, j)}+(-1)^{j} B_{:, \sigma(z, 1)} .
$$

(5) Delete column $B_{:, \sigma(z, 1)}$ for each $z \in \mathcal{S}_{h}$.

The stiffness matrix for the Stokes problem based on the $\stackrel{\circ}{h}^{\mathrm{PS}} \times \hat{Y}_{h}^{\mathrm{PS}}$ pair is then given by

$$
\left(\begin{array}{cc}
A & B \\
B^{\top} & 0
\end{array}\right)
$$

\section{Implemenation aspects for Worsey Farin Splits}

6.1. A basis for $\hat{Y}_{h}^{\mathrm{WF}}$ and the construction of the algebraic system. Notice that the collection of local triangulations $\mathcal{T}_{e}$ (with $e \in \mathcal{E}_{h}^{\mathcal{S}}$ ) do not form a disjoint partition of the global triangulation $\mathcal{T}_{h}^{\mathrm{WF}}$. In particular, there exists $K \in \mathcal{T}_{h}^{\mathrm{WF}}$ such that $K \in \mathcal{T}_{e}$ and $K \in \mathcal{T}_{s}$ with $e, s \in \mathcal{E}_{h}^{\mathcal{S}}$ and $e \neq s$. As a result, the methodology used in the previous section for Powell-Sabin meshes is not directly applicable.

Instead, we consider a geometric decomposition of the mesh based on the face split points in $\mathcal{T}_{h}^{\mathrm{WF}}$. To this end, we denote by $\mathcal{S}_{h}^{I}$ and $\mathcal{S}_{h}^{B}$ the sets of interior and boundary face split points, respectively, and set $\mathcal{S}_{h}=\mathcal{S}_{h}^{I} \cup \mathcal{S}_{h}^{B}$. For $z \in \mathcal{S}_{h}$, let $\mathcal{T}_{z}:=\left\{K_{z}^{(1)}, \ldots, K_{z}^{\left(n_{z}\right)}\right\}$ denote the set of tetrahedra in $\mathcal{T}_{h}^{\mathrm{WF}}$ that have $z$ as a vertex. Here, $n_{z}=6$ if $z$ is an interior vertex, and $n_{z}=3$ if $z$ is a boundary vertex. For an interior face split point $z$, we assume the simplices in $\mathcal{T}_{z}$ are labeled such that

$$
K_{z}^{(1)}, K_{z}^{(2)}, K_{z}^{(3)} \subset T^{(1)}, \quad K_{z}^{(4)}, K_{z}^{(5)}, K_{z}^{(6)} \subset T^{(2)}
$$


for some $T^{(1)}, T^{(2)} \in \mathcal{T}_{h}$, and that $K_{z}^{(j)}$ and $K_{z}^{(j+3)}$ share a common face for $j \in\{1,2,3\}$. For a boundary split point $z$, the set $\mathcal{T}_{z}=\left\{K_{z}^{(1)}, K_{z}^{(2)}, K_{z}^{(3)}\right\}$ is labeled arbitrarily.

We clearly have

$$
\mathcal{T}_{h}^{\mathrm{WF}}=\left\{K_{z}^{(j)}: z \in \mathcal{S}_{h}, j=1, \ldots, n_{z}\right\},
$$

and the map $(z, j) \rightarrow K_{z}^{(j)}$ is injective. Furthermore, each local partition $\mathcal{T}_{z}$ contains three singular edges.

Proposition 6.1. There holds

$$
\operatorname{dim} \hat{Y}_{h}^{\mathrm{WF}}=4\left|\mathcal{S}_{h}^{I}\right|+\left|\mathcal{S}_{h}^{B}\right|
$$

Proof. By Proposition 4.5, we have

$$
\operatorname{dim} \hat{Y}_{h}^{\mathrm{WF}}=4\left|\mathcal{T}_{h}\right|+2\left|\mathcal{F}_{h}^{I}\right|,
$$

where $\left|\mathcal{F}_{h}^{I}\right|$ is the number of interior faces in $\mathcal{T}_{h}$. From (6.1), we have

$$
12\left|\mathcal{T}_{h}\right|=\left|\mathcal{T}_{h}^{\mathrm{WF}}\right|=6\left|\mathcal{S}_{h}^{I}\right|+3\left|\mathcal{S}_{h}^{B}\right|,
$$

and by construction of the Worsey-Farin split, there holds

$$
\left|\mathcal{F}_{h}\right|=\left|\mathcal{S}_{h}^{I}\right| .
$$

Therefore,

$$
\operatorname{dim} \hat{Y}_{h}^{\mathrm{WF}}=4\left|\mathcal{T}_{h}\right|+2\left|\mathcal{F}_{h}^{I}\right|=\frac{1}{3}\left(6\left|\mathcal{S}_{h}^{I}\right|+3\left|\mathcal{S}_{h}^{B}\right|\right)+2\left|\mathcal{S}_{h}^{I}\right|=4\left|\mathcal{S}_{h}^{I}\right|+\left|\mathcal{S}_{h}^{B}\right|
$$

For an interior split point $z$, and for a piecewise constant function $q$ on $\mathcal{T}_{z}$, the three constraints $\theta_{e}(q)=0 \mathrm{read}$

$$
\begin{aligned}
& q_{1}-q_{2}+q_{5}-q_{4}=0, \\
& q_{2}-q_{3}+q_{6}-q_{5}=0, \\
& q_{3}-q_{1}+q_{4}-q_{6}=0,
\end{aligned}
$$

where $q_{j}=\left.q\right|_{K_{z}^{(j)}}$

We write this as a $3 \times 6$ linear system

$$
C \vec{q}:=\left(\begin{array}{cccccc}
1 & -1 & 0 & -1 & 1 & 0 \\
0 & 1 & -1 & 0 & -1 & 1 \\
-1 & 0 & 1 & 1 & 0 & -1
\end{array}\right)\left(\begin{array}{l}
q_{1} \\
q_{2} \\
q_{3} \\
q_{4} \\
q_{5} \\
q_{6}
\end{array}\right)=0 .
$$

We clearly see that this matrix has rank 2 (e.g., adding the first and third rows gets the negation of the second row). We find that the nullspace of $C$ is given by

$$
\operatorname{null}(C)=\operatorname{span}\left\{\left(\begin{array}{l}
1 \\
1 \\
1 \\
0 \\
0 \\
0
\end{array}\right),\left(\begin{array}{l}
1 \\
0 \\
0 \\
1 \\
0 \\
0
\end{array}\right),\left(\begin{array}{l}
0 \\
1 \\
0 \\
0 \\
1 \\
0
\end{array}\right),\left(\begin{array}{c}
-1 \\
-1 \\
0 \\
0 \\
0 \\
1
\end{array}\right)\right\} .
$$

These four vectors implicitly give us a basis for $\hat{Y}_{h}^{\mathrm{WF}}$. In particular, we have 
Proposition 6.2. For $z \in \mathcal{S}_{h}$ and $j \in\left\{1,2, \ldots, n_{z}\right\}$, let $\varphi_{z}^{(j)}$ be the piecewise constant function

$$
\left.\varphi_{z}^{(j)}\right|_{K_{v}^{(i)}}=\delta_{v, z} \delta_{i, j} \quad \forall v, z \in \mathcal{S}_{h}, i=1, \ldots, n_{v}, j=1, \ldots, n_{z} .
$$

For an interior face split point $z$, define

$$
\begin{aligned}
& \psi_{z}^{(3)}=\varphi_{z}^{(3)}+\varphi_{z}^{(1)}+\varphi_{z}^{(2)}, \\
& \psi_{z}^{(4)}=\varphi_{z}^{(4)}+\varphi_{z}^{(1)}, \\
& \psi_{z}^{(5)}=\varphi_{z}^{(5)}+\varphi_{z}^{(2)}, \\
& \psi_{z}^{(6)}=\varphi_{z}^{(6)}-\varphi_{z}^{(1)}-\varphi_{z}^{(2)} .
\end{aligned}
$$

For a boundary face split point $z$, define

$$
\psi_{z}^{(3)}=\varphi_{z}^{(3)}+\varphi_{z}^{(1)}+\varphi_{z}^{(2)} .
$$

Then $\left\{\psi_{z}^{(j)}\right\}$ is a basis of $\hat{Y}_{h}^{\mathrm{WF}}$.

Proof. The proof essentially follows from the same arguments as Proposition [5.1, noting that the number of given $\psi_{z}^{(j)}$ is

$$
4\left|\mathcal{S}_{h}^{I}\right|+\left|\mathcal{S}_{h}^{B}\right|=\operatorname{dim} \hat{Y}_{h}^{\mathrm{WF}}
$$

by Proposition 6.1

As in the two-dimensional case, Proposition 6.2 give an algorithm to construct the stiffness matrix for the Stokes problem using the $\dot{\boldsymbol{V}}_{h}^{\mathrm{WF}} \times \hat{Y}_{h}^{\mathrm{WF}}$ pair. First, we construct the stiffness matrix based on the $\stackrel{\circ}{h}^{\mathrm{WF}} \times \mathcal{P}_{0}\left(\mathcal{T}_{h}^{\mathrm{WF}}\right)$ pair:

$$
\left(\begin{array}{cc}
A & \tilde{B} \\
\tilde{B}^{\top} & 0
\end{array}\right)
$$

and then perform elementary column operations on the $\tilde{B}$.

Let $\left\{\phi^{(k)}\right\}_{k=1}^{N}$ denote a basis of $\dot{V}_{h}^{\mathrm{WF}}$ with $N=\operatorname{dim} \boldsymbol{V}_{h}^{\mathrm{PS}}$ Let $M=\operatorname{dim} \mathcal{P}_{0}\left(\mathcal{T}_{h}^{\mathrm{PS}}\right)$, the number of tetrahedra in $\mathcal{T}_{h}^{\mathrm{WF}}$, and introduce the local-to-global label mapping $\sigma: \mathcal{S}_{h} \times\left\{1, \ldots, n_{z}\right\} \rightarrow$ $\{1,2, \ldots, M\}$ such that

$$
\tilde{B}_{k, \sigma(z, j)}=-\int_{\Omega}\left(\operatorname{div} \phi^{(k)}\right) \varphi_{z}^{(j)}
$$

\section{Algorithm 1}

(1) Construct Worsey-Farin triangulation $\mathcal{T}_{h}^{\text {PS }}$

(2) Construct $\tilde{B} \in \mathbb{R}^{N \times M}$ based on the $\stackrel{\circ}{h}^{\mathrm{WF}} \times \mathcal{P}_{0}\left(\mathcal{T}_{h}^{\mathrm{WF}}\right)$ pair.

(3) Set $B=\tilde{B}$.

(4) For each interior face split point $z \in \mathcal{S}_{h}$ do the elementary column operations

$$
\begin{aligned}
& B_{:, \sigma(z, 3)}=B_{:, \sigma(z, 3)}+B_{:, \sigma(z, 1)}+B_{:, \sigma(z, 2)}, \\
& B_{:, \sigma(z, 4)}=B_{:, \sigma(z, 4)}+B_{:, \sigma(z, 1)} \\
& B_{:, \sigma(z, 5)}=B_{:, \sigma(z, 5)}+B_{:, \sigma(z, 2)}, \\
& B_{:, \sigma(z, 6)}=B_{:, \sigma(z, 6)}-B_{:, \sigma(z, 1)}-B_{:, \sigma(z, 2)} .
\end{aligned}
$$

(5) For each boundary face split point $z \in \mathcal{S}_{h}$ do the elementary column operation

$$
B_{:, \sigma(z, 3)}=B_{:, \sigma(z, 3)}+B_{:, \sigma(z, 1)}+B_{:, \sigma(z, 2)} .
$$

(6) Delete columns $B_{:, \sigma(z, 1)}$ and $B_{:, \sigma(z, 2)}$ for each $z \in \mathcal{S}_{h}$. 
TABLE 1. Errors and rates of convergence for example (7.3) with $\nu=1$.

\begin{tabular}{||ccccccc||}
\hline$h$ & $\left\|\boldsymbol{u}-\boldsymbol{u}_{h}\right\|_{L^{2}(\Omega)}$ & rate & $\left\|p-p_{h}\right\|_{L^{2}(\Omega)}$ & rate & $\left\|\nabla \cdot \boldsymbol{u}_{h}\right\|_{L^{2}(\Omega)}$ & $\beta$ \\
\hline $2^{-2}$ & $1.70 \mathrm{E}-01$ & - & $5.26 \mathrm{E} 00$ & - & $2.70 \mathrm{E}-14$ & $1.56 \mathrm{E}-01$ \\
$2^{-3}$ & $5.66 \mathrm{E}-02$ & 1.587 & $3.77 \mathrm{E} 00$ & 0.480 & $6.65 \mathrm{E}-14$ & $1.38 \mathrm{E}-01$ \\
$2^{-4}$ & $1.35 \mathrm{E}-02$ & 2.068 & $1.68 \mathrm{E} 00$ & 1.166 & $2.38 \mathrm{E}-13$ & $1.07 \mathrm{E}-01$ \\
$2^{-5}$ & $3.35 \mathrm{E}-03$ & 2.011 & $8.28 \mathrm{E}-01$ & 1.021 & $8.38 \mathrm{E}-12$ & $1.06 \mathrm{E}-01$ \\
$2^{-6}$ & $8.77 \mathrm{E}-04$ & 1.934 & $4.25 \mathrm{E}-01$ & 0.962 & $4.05 \mathrm{E}-10$ & $9.34 \mathrm{E}-02$ \\
\hline
\end{tabular}

The stiffness matrix for the Stokes problem based on the $\stackrel{\circ}{h}_{h}^{\mathrm{WF}} \times \hat{Y}_{h}^{\mathrm{WF}}$ pair is then given by

$$
\left(\begin{array}{cc}
A & B \\
B^{\top} & 0
\end{array}\right)
$$

\section{NUMERICAL EXPERIMENTS}

In this section, we perform some simple numerical experiments for the Stokes problem on PowellSabin and Worsey-Farin splits. We note standard theory shows that the velocity and pressure errors satisfy

$$
\begin{aligned}
\left|\boldsymbol{u}-\boldsymbol{u}_{h}\right|_{H^{1}(\Omega)} & \leq\left(1+\beta^{-1}\right) \inf _{\boldsymbol{v}_{h} \in \boldsymbol{V}_{h}}\left|\boldsymbol{v}_{h}-\boldsymbol{u}\right|_{H^{1}(\Omega)}, \\
\left\|p-p_{h}\right\|_{L^{2}(\Omega)} & \leq \inf _{q \in Y_{h}}\|p-q\|_{L^{2}(\Omega)}+\frac{\nu}{\beta}\left|\boldsymbol{u}-\boldsymbol{u}_{h}\right|_{H^{1}(\Omega)},
\end{aligned}
$$

where either $\boldsymbol{V}_{h} \times Y_{h}=\boldsymbol{V}_{h}^{\mathrm{PS}} \times \stackrel{\circ}{Y}_{h}^{\mathrm{PS}}$ or $\boldsymbol{V}_{h} \times Y_{h}=\stackrel{\circ}{\boldsymbol{V}}_{h}^{\mathrm{WF}} \times \stackrel{\circ}{Y}_{h}^{\mathrm{WF}}, \nu>0$ is the viscosity, and $\beta$ is the inf-sup constant for the finite element pair $\boldsymbol{V}_{h} \times Y_{h}$.

7.1. The Stokes pair on Powell-Sabin Splits. We consider the example such that the data is taken to be $\Omega=(0,1)^{2}$, and the source function is chosen such that the exact velocity and pressure solutions for (2.1) are given respectively as

$$
\boldsymbol{u}=\left(\begin{array}{c}
\pi \sin ^{2}\left(\pi x_{1}\right) \sin \left(2 \pi x_{2}\right) \\
-\pi \sin ^{2}\left(\pi x_{2}\right) \sin \left(2 \pi x_{1}\right)
\end{array}\right), \quad p=\cos \left(\pi x_{1}\right) \cos \left(\pi x_{2}\right) .
$$

Let $\mathcal{T}_{h}$ be a Delaunay triangulation of $\Omega$ and $\mathcal{T}_{h}^{P S}$ the corresponding Powell-Sabin global triangulation.

The resulting errors, rates of convergence, and inf-sup constants are listed in Tables 11 and 2 for viscosities $\nu=1$ and $\nu=10^{-2}$, respectively. The results show that the $L^{2}$ pressure error and the $H^{1}$ velocity error converge with linear rate, the discrete velocity solution (and error) are independent of the viscosity $\nu$, and the pressure error improves for small viscosity. The experiments also show that the inf-sup constant does not deteriorate as the mesh is refined with $\beta \approx 0.1$. These results are in agreement with the theoretical estimates (7.1)-(7.2)

In Tables 3 and 4 we compute the right-hand side of (7.2) and (7.1), respectively, and compare the data with the computed errors $\left\|p-p_{h}\right\|_{L^{2}(\Omega)}$ and $\left|\boldsymbol{u}-\boldsymbol{u}_{h}\right|_{H^{1}(\Omega)}$. Again, the results are consistent with (7.1) - (7.2), and they suggest that the term $\left|\boldsymbol{u}-\boldsymbol{u}_{h}\right|_{H^{1}(\Omega)}$ is the dominant term in the pressure error (7.2).

7.2. The Stokes pair on Worsey-Farin Splits. We consider the example such that the data is taken to be $\Omega=(0,1)^{3}$, and the source function is chosen such that the exact velocity and pressure solutions for (2.1) are given respectively as

$$
\boldsymbol{u}=\left(\begin{array}{c}
\pi \sin ^{2}\left(\pi x_{1}\right) \sin \left(2 \pi x_{2}\right) \\
-\pi \sin ^{2}\left(\pi x_{2}\right) \sin \left(2 \pi x_{1}\right) \\
0
\end{array}\right), \quad p=\cos \left(\pi x_{1}\right) \cos \left(\pi x_{2}\right) \cos \left(\pi x_{3}\right) .
$$


TABLE 2. Errors and rates of convergence for example (7.3) with $\nu=10^{-2}$.

\begin{tabular}{||cccccc||}
\hline$h$ & $\left\|\boldsymbol{u}-\boldsymbol{u}_{h}\right\|_{L^{2}(\Omega)}$ & rate & $\left\|p-p_{h}\right\|_{L^{2}(\Omega)}$ & rate & $\left\|\nabla \cdot \boldsymbol{u}_{h}\right\|_{L^{2}(\Omega)}$ \\
\hline $2^{-2}$ & $1.70 \mathrm{E}-01$ & - & $1.02 \mathrm{E}-01$ & - & $2.43 \mathrm{E}-14$ \\
$2^{-3}$ & $5.66 \mathrm{E}-02$ & 1.587 & $5.79 \mathrm{E}-02$ & 0.816 & $5.88 \mathrm{E}-14$ \\
$2^{-4}$ & $1.35 \mathrm{E}-02$ & 2.068 & $2.76 \mathrm{E}-02$ & 1.069 & $2.36 \mathrm{E}-13$ \\
$2^{-5}$ & $3.35 \mathrm{E}-03$ & 2.011 & $1.37 \mathrm{E}-02$ & 1.010 & $8.39 \mathrm{E}-12$ \\
$2^{-6}$ & $8.77 \mathrm{E}-04$ & 1.934 & $6.96 \mathrm{E}-03$ & 0.977 & $4.05 \mathrm{E}-10$ \\
\hline
\end{tabular}

TABLE 3. Errors for example (7.3) with $\nu=10^{-2}$ and the RHS of (7.2) .

\begin{tabular}{||cccccc||}
\hline$h$ & $\left\|p-p_{h}\right\|_{L^{2}(\Omega)}$ & $\left|\boldsymbol{u}-\boldsymbol{u}_{h}\right|_{H^{1}(\Omega)}$ & $\beta$ & $\inf _{q \in \dot{Y}_{h}^{\mathrm{PS}}}\|p-q\|_{L^{2}(\Omega)}$ & RHS of (7.2) \\
\hline $2^{-2}$ & $1.02 \mathrm{E}-01$ & $3.77 \mathrm{E} 00$ & $1.56 \mathrm{E}-01$ & $6.08 \mathrm{E}-02$ & $3.02 \mathrm{E}-01$ \\
$2^{-3}$ & $5.79 \mathrm{E}-02$ & $2.17 \mathrm{E} 00$ & $1.38 \mathrm{E}-01$ & $2.77 \mathrm{E}-02$ & $1.84 \mathrm{E}-01$ \\
$2^{-4}$ & $2.76 \mathrm{E}-02$ & $1.07 \mathrm{E} 00$ & $1.07 \mathrm{E}-01$ & $1.35 \mathrm{E}-02$ & $1.13 \mathrm{E}-01$ \\
$2^{-5}$ & $1.37 \mathrm{E}-02$ & $5.32 \mathrm{E}-01$ & $1.06 \mathrm{E}-01$ & $6.61 \mathrm{E}-03$ & $5.67 \mathrm{E}-02$ \\
$2^{-6}$ & $6.96 \mathrm{E}-03$ & $2.72 \mathrm{E}-01$ & $9.34 \mathrm{E}-02$ & $3.28 \mathrm{E}-03$ & $3.24 \mathrm{E}-02$ \\
\hline
\end{tabular}

TABLE 4. Errors for example (7.3) with $\nu=10^{-2}$ and the RHS of (7.1) .

\begin{tabular}{||ccccc||}
\hline$h$ & $\left|\boldsymbol{u}-\boldsymbol{u}_{h}\right|_{H^{1}(\Omega)}$ & $\beta$ & $\inf _{\boldsymbol{v}_{h} \in \boldsymbol{V}_{h}^{\mathrm{PS}}}\left|\boldsymbol{v}_{h}-\boldsymbol{u}\right|_{H^{1}(\Omega)}$ & RHS of (7.1) \\
\hline $2^{-2}$ & $3.77 \mathrm{E} 00$ & $1.56 \mathrm{E}-01$ & $3.08 \mathrm{E} 00$ & $2.28 \mathrm{E}+01$ \\
$2^{-3}$ & $2.17 \mathrm{E} 00$ & $1.38 \mathrm{E}-01$ & $1.63 \mathrm{E} 00$ & $1.34 \mathrm{E}+01$ \\
$2^{-4}$ & $1.07 \mathrm{E} 00$ & $1.07 \mathrm{E}-01$ & $8.04 \mathrm{E}-01$ & $8.31 \mathrm{E} 00$ \\
$2^{-5}$ & $5.32 \mathrm{E}-01$ & $1.06 \mathrm{E}-01$ & $4.06 \mathrm{E}-01$ & $4.23 \mathrm{E} 00$ \\
$2^{-6}$ & $2.72 \mathrm{E}-01$ & $9.34 \mathrm{E}-02$ & $2.05 \mathrm{E}-01$ & $2.39 \mathrm{E} 00$ \\
\hline
\end{tabular}

TABLE 5. Errors and rates of convergence for example (7.4) with $\nu=1$.

\begin{tabular}{||ccccccc||}
\hline$h$ & $\left\|\boldsymbol{u}-\boldsymbol{u}_{h}\right\|_{L^{2}(\Omega)}$ & rate & $\left\|p-p_{h}\right\|_{L^{2}(\Omega)}$ & rate & $\left\|\nabla \cdot \boldsymbol{u}_{h}\right\|_{L^{2}(\Omega)}$ & $\beta$ \\
\hline $1 / 2$ & $1.29 \mathrm{E} 00$ & - & $9.81 \mathrm{E} 00$ & - & $5.07 \mathrm{E}-14$ & $1.31 \mathrm{E}-01$ \\
$1 / 4$ & $8.58 \mathrm{E}-01$ & 0.588 & $19.4 \mathrm{E} 00$ & -0.98 & $5.20 \mathrm{E}-13$ & $1.31 \mathrm{E}-01$ \\
$1 / 8$ & $3.93 \mathrm{E}-01$ & 1.286 & $16.6 \mathrm{E} 00$ & 0.414 & $2.68 \mathrm{E}-12$ & $1.32 \mathrm{E}-01$ \\
$1 / 16$ & $1.32 \mathrm{E}-01$ & 1.573 & $10.5 \mathrm{E} 00$ & 0.667 & $4.10 \mathrm{E}-12$ & $1.32 \mathrm{E}-01$ \\
$1 / 32$ & $3.69 \mathrm{E}-02$ & 1.839 & $5.75 \mathrm{E} 00$ & 0.872 & $4.32 \mathrm{E}-12$ & $1.32 \mathrm{E}-01$ \\
$1 / 48$ & $1.68 \mathrm{E}-02$ & 1.941 & $3.93 \mathrm{E} 00$ & 0.936 & $6.07 \mathrm{E}-12$ & $1.32 \mathrm{E}-01$ \\
\hline
\end{tabular}

Let $\mathcal{T}_{h}$ be a Delaunay triangulation of $\Omega$ and $\mathcal{T}_{h}^{W F}$ be the corresponding Worsey-Farin global triangulation.

The resulting rates of convergence of the numerical experiments for viscosities $\nu=1$ and $\nu=10^{-3}$ are listed in Tables 5 and [6, respectively. We also state the computed inf-sup constant on these meshes, and the results show that that it stays uniformly bounded from below with $\beta \approx 0.13$ on all meshes. The stated errors, especially those in Table 5, indicate that the rates of convergence are still in the preasymptotic regime. On the other hand, for small viscosity value $\nu=10^{-3}$, Table 6 shows that the pressure error converges with linear rate. This behavior suggests that the velocity error is the dominating term in (7.2).

To verify this claim, we explicitly compute the right-hand side of (7.2) and (7.1) and report the results in Tables 7 and 8 , respectively. The results show that indeed $\left|\boldsymbol{u}-\boldsymbol{u}_{h}\right|_{H^{1}(\Omega)}$ is the dominating term in the pressure error (7.2). 
TABLE 6. Errors and rates of convergence for example (7.4) with $\nu=10^{-3}$.

\begin{tabular}{||cccccc||}
\hline$h$ & $\left\|\boldsymbol{u}-\boldsymbol{u}_{h}\right\|_{L^{2}(\Omega)}$ & rate & $\left\|p-p_{h}\right\|_{L^{2}(\Omega)}$ & rate & $\left\|\nabla \cdot \boldsymbol{u}_{h}\right\|_{L^{2}(\Omega)}$ \\
\hline $1 / 2$ & $1.29 \mathrm{E} 00$ & - & $1.33 \mathrm{E}-01$ & - & $1.28 \mathrm{E}-15$ \\
$1 / 4$ & $8.58 \mathrm{E}-01$ & 0.588 & $6.97 \mathrm{E}-02$ & 0.932 & $3.43 \mathrm{E}-14$ \\
$1 / 8$ & $3.93 \mathrm{E}-01$ & 1.286 & $3.70 \mathrm{E}-02$ & 0.911 & $3.22 \mathrm{E}-13$ \\
$1 / 16$ & $1.32 \mathrm{E}-01$ & 1.574 & $1.91 \mathrm{E}-02$ & 0.953 & $6.40 \mathrm{E}-13$ \\
$1 / 32$ & $3.69 \mathrm{E}-02$ & 1.838 & $9.68 \mathrm{E}-03$ & 0.980 & $9.52 \mathrm{E}-13$ \\
$1 / 48$ & $9.63 \mathrm{E}-03$ & 1.940 & $4.89 \mathrm{E}-03$ & 0.983 & $1.03 \mathrm{E}-12$ \\
\hline
\end{tabular}

TABLE 7. Errors for example (7.4) with $\nu=10^{-3}$ and the RHS of (7.2) .

\begin{tabular}{||cccccc||}
\hline$h$ & $\left\|p-p_{h}\right\|_{L^{2}(\Omega)}$ & $\left|\boldsymbol{u}-\boldsymbol{u}_{h}\right|_{H^{1}(\Omega)}$ & $\beta$ & $\inf _{q \in \dot{Y}_{h}^{\mathrm{WF}}}\|p-q\|_{L^{2}(\Omega)}$ & RHS of (7.2) \\
\hline $1 / 2$ & $1.33 \mathrm{E}-01$ & $1.07 \mathrm{E}+01$ & $1.31 \mathrm{E}-01$ & $5.00 \mathrm{E}-01$ & $5.81 \mathrm{E}-01$ \\
$1 / 4$ & $6.97 \mathrm{E}-02$ & $8.50 \mathrm{E} 00$ & $1.31 \mathrm{E}-01$ & $6.70 \mathrm{E}-02$ & $1.31 \mathrm{E}-01$ \\
$1 / 6$ & $4.81 \mathrm{E}-02$ & $6.82 \mathrm{E} 00$ & $1.32 \mathrm{E}-01$ & $4.43 \mathrm{E}-02$ & $9.59 \mathrm{E}-02$ \\
$1 / 8$ & $3.70 \mathrm{E}-02$ & $5.60 \mathrm{E} 00$ & $1.32 \mathrm{E}-01$ & $3.30 \mathrm{E}-02$ & $7.54 \mathrm{E}-02$ \\
$1 / 10$ & $3.02 \mathrm{E}-02$ & $4.71 \mathrm{E} 00$ & $1.32 \mathrm{E}-01$ & $2.63 \mathrm{E}-02$ & $6.19 \mathrm{E}-02$ \\
$1 / 12$ & $2.55 \mathrm{E}-02$ & $4.06 \mathrm{E} 00$ & $1.32 \mathrm{E}-01$ & $2.19 \mathrm{E}-02$ & $5.26 \mathrm{E}-02$ \\
\hline
\end{tabular}

TABLE 8. Errors for example (7.4) with $\nu=10^{-3}$ and the RHS of (7.1) .

\begin{tabular}{||ccccc||}
\hline$h$ & $\left|\boldsymbol{u}-\boldsymbol{u}_{h}\right|_{H^{1}(\Omega)}$ & $\beta$ & inf $_{\boldsymbol{v}_{h} \in \dot{\boldsymbol{V}}_{h}^{\mathrm{WF}}}\left|\boldsymbol{v}_{h}-\boldsymbol{u}\right|_{H^{1}(\Omega)}$ & $\mathrm{RHS}$ of $(\mathbf{7 . 1})$ \\
\hline $1 / 2$ & $1.07 \mathrm{E}+01$ & $1.31 \mathrm{E}-01$ & $9.99 \mathrm{E} 00$ & $8.62 \mathrm{E}+01$ \\
$1 / 4$ & $8.50 \mathrm{E} 00$ & $1.31 \mathrm{E}-01$ & $5.85 \mathrm{E} 00$ & $5.05 \mathrm{E}+01$ \\
$1 / 6$ & $6.82 \mathrm{E} 00$ & $1.32 \mathrm{E}-01$ & $4.08 \mathrm{E} 00$ & $3.49 \mathrm{E}+01$ \\
$1 / 8$ & $5.60 \mathrm{E} 00$ & $1.32 \mathrm{E}-01$ & $3.10 \mathrm{E} 00$ & $2.65 \mathrm{E}+01$ \\
$1 / 10$ & $4.71 \mathrm{E} 00$ & $1.32 \mathrm{E}-01$ & $2.49 \mathrm{E} 00$ & $2.13 \mathrm{E}+01$ \\
$1 / 12$ & $4.06 \mathrm{E} 00$ & $1.32 \mathrm{E}-01$ & $2.08 \mathrm{E} 00$ & $1.78 \mathrm{E}+01$ \\
\hline
\end{tabular}

7.3. Iterated Penalty Method for $\left(\mathbf{P}_{\mathbf{1}}, \mathbf{P}_{\mathbf{0}}\right)$ pair on Worsey-Farin Splits. We consider the example such that the data is taken to be $\Omega=(0,1)^{3}$, and the source function is chosen such that the exact velocity and pressure solutions for (2.1) are given respectively as

$$
\boldsymbol{u}(x, y, z)=\nabla \times\left(\begin{array}{l}
0 \\
g \\
g
\end{array}\right), \quad p=\frac{1}{9} \frac{\partial^{2} g}{\partial x \partial y}
$$

where

$$
g=g(x, y, z)=2^{12}\left(x-x^{2}\right)^{2}\left(y-y^{2}\right)^{2}\left(z-z^{2}\right)^{2} .
$$

Similar to the previous section, we let $\mathcal{T}_{h}$ be a Delaunay triangulation of $\Omega$ and $\mathcal{T}_{h}^{\mathrm{WF}}$ be the corresponding Worsey-Farin global triangulation.

The iterated penalty method [3] applied to the Stokes equations with $\stackrel{\circ}{h}_{h}=\dot{\boldsymbol{V}}_{h}^{\mathrm{WF}}$ reads : Let $\boldsymbol{u}_{h}^{0}=\mathbf{0}$ and $\rho, \gamma>0$ be parameters. For $n \geq 1, \boldsymbol{u}_{h}^{n}$ is recursively defined to be the solution to the variational formulation

$$
\nu\left(\nabla \boldsymbol{u}_{h}^{n}, \nabla \boldsymbol{v}\right)+\gamma\left(\nabla \cdot \boldsymbol{v}, \nabla \cdot \boldsymbol{u}_{h}^{n}\right)=(\boldsymbol{f}, \boldsymbol{v})-\left(\sum_{i=0}^{n-1} \rho \nabla \cdot \boldsymbol{u}_{h}^{i}, \nabla \cdot \boldsymbol{v}\right), \quad \forall \boldsymbol{v} \in \stackrel{\circ}{V}_{h}^{\mathrm{WF}}
$$


TABLE 9. Errors and rates of convergence for example (7.5) with $\nu=1$.

\begin{tabular}{||ccccccc||}
\hline$h$ & $\left\|\boldsymbol{u}-\boldsymbol{u}_{h}^{n}\right\|_{L^{2}(\Omega)}$ & rate & $\left|\boldsymbol{u}-\boldsymbol{u}_{h}^{n}\right|_{H^{1}(\Omega)}$ & rate & $\left\|p-p_{h}^{n}\right\|_{L^{2}(\Omega)}$ & rate \\
\hline $2^{-2}$ & 1.11768 & - & 11.55063 & - & 25.32256 & - \\
$2^{-3}$ & 0.48896 & 1.19273 & 7.53829 & 0.61566 & 22.35349 & 0.17992 \\
$2^{-4}$ & 0.15482 & 1.65908 & 4.15598 & 0.85905 & 13.67635 & 0.70882 \\
$2^{-5}$ & 0.04176 & 1.89040 & 2.13224 & 0.96282 & 7.24129 & 0.91736 \\
$1 / 48$ & 0.01881 & 1.96680 & 1.42643 & 0.99145 & 4.88909 & 0.96875 \\
\hline
\end{tabular}

It is shown in [3] that $\lim _{n \rightarrow \infty} \boldsymbol{u}_{h}^{n}=\boldsymbol{u}_{h}$ and $\lim _{n \rightarrow \infty} \sum_{i=0}^{n} \rho \nabla \cdot \boldsymbol{u}_{h}^{i}=p_{h}$. Also, [3] suggests to use $\left\|\nabla \cdot \boldsymbol{u}_{h}^{n}\right\|_{L^{2}(\Omega)}$ as a stopping criterion since the difference error between $\boldsymbol{u}_{h}^{n}, \boldsymbol{u}_{h}$ is given by

$$
\left\|\boldsymbol{u}_{h}^{n}-\boldsymbol{u}_{h}\right\|_{L^{2}(\Omega)} \leq C\left\|\nabla \cdot \boldsymbol{u}_{h}^{n}\right\|_{L^{2}(\Omega)} .
$$

The resulting rates of convergence of the numerical experiment are listed in Tables 9 . The errors $\left\|\boldsymbol{u}-\boldsymbol{u}_{h}^{n}\right\|_{L^{2}(\Omega)}$ and $\left\|p-p_{h}^{n}\right\|_{L^{2}(\Omega)}$ are computed with $\left\|\nabla \cdot \boldsymbol{u}_{h}^{n}\right\|_{L^{2}(\Omega)} \leq 10^{-7}$ and $\gamma=\rho=100$.

In Table 10 we provide a time comparison between the (IPM) and the method described in Algorithm 1. All of the timings were done on a machine with a single $3.60 \mathrm{GHz}$ Intel Core i9-9900K processor with $128 \mathrm{~GB}$ of $2400 \mathrm{MHz}$ DDR4 memory.

For the iterated penalty method, we select $\gamma=\rho=100$ and terminate iterations once $\| \nabla$. $\boldsymbol{u}_{h}^{n} \|_{L^{2}(\Omega)} \leq 10^{-7}$. At each iteration of IPM, equation (7.6) must be resolved. In our work, this vector Poisson problem type problem is solved via a conjugate gradient method with an algebraic multigrid (AMG) preconditioner. It is well known that optimal multigrid methods can be designed for this class of problems [17.

For Algorithm 1, we instead work with the full discretization matrix (6.2), which results in a symmetric indefinite linear system. To efficiently solve this saddle point problem, a block preconditioned Krylov subspace method is used [16]. In particular, we precondition the stiffness matrix (6.2) by the block diagonal matrix

$$
\left(\begin{array}{ll}
A & 0 \\
0 & S
\end{array}\right)
$$

where $S=B^{\top} A^{-1} B$ is the Schur complement, and the flexible GMRES method as an outer iteration. The solver is terminated once the Euclidean norm of the residual is less than or equal to $10^{-8}$. For simplicity, the inner preconditioners are as follows: we use a preconditioner to $A$ for approximating $A^{-1}$, and the Schur complement is approximated as $S \approx B^{\top} \operatorname{diag}\left(A^{-1}\right) B$. It should be noted that this is not the only choice for block-type preconditioning of the Stokes problem (e.g., see [4]); however, our numerical experiments indicate that we can repurpose existing preconditioners to efficiently solve linear systems that arise from the proposed finite element discretization.

From Table 10, it is evident that for a fixed mesh spacing, the total run times for both methods are competitive, with the IPM technique being slightly slower for larger meshes. We find that in the case of the linear Stokes problem, by utilizing preexisting preconditioners, both approaches provide similar accuracy and time to solution metrics.

To obtain a Reynolds robust, optimally scaling preconditioner, special multigrid techniques may have to be investigated [6, 5].

\section{REFERENCES}

[1] D. N. Arnold and J. Qin. Quadratic velocity/linear pressure Stokes elements. Advances in computer methods for partial differential equations, 7:28-34, 1992.

[2] D. Boffi, J. Guzman, and M. Neilan. Convergence of Lagrange finite elements for the Maxwell eigenvalue problem in 2d. arXiv preprint arXiv:2003.08381, 2020.

[3] S. C. Brenner and L. Scott. The Mathematical Theory of Finite Elements Methods, volume 3. Springer, 2007.

[4] H. Elman, V. Howle, J. Shadid, R. Shuttleworth, and R. Tuminaro. A taxonomy and comparison of parallel block multi-level preconditioners for the incompressible Navier-Stokes equations. Journal of Computational Physics, 227(3):1790-1808, 2008. 
TABLE 10. Time comparison between (IPM) and Algorithm 1 for example (7.5) with $\nu=1$.

\begin{tabular}{||ccc||}
\hline$h$ & $(\mathrm{IPM})$ & Algorithm 1 \\
\hline $2^{-2}$ & $5.08 \mathrm{E}+00$ & $3.35 \mathrm{E}+00$ \\
$2^{-3}$ & $1.68 \mathrm{E}+01$ & $2.93 \mathrm{E}+01$ \\
$2^{-4}$ & $4.80 \mathrm{E}+02$ & $2.60 \mathrm{E}+02$ \\
$2^{-5}$ & $2.39 \mathrm{E}+03$ & $9.37 \mathrm{E}+02$ \\
$1 / 48$ & $7.31 \mathrm{E}+03$ & $6.45 \mathrm{E}+03$ \\
\hline
\end{tabular}

[5] P. E. Farrell, M. G. Knepley, L. Mitchell, and F. Wechsung. Pcpatch: software for the topological construction of multigrid relaxation methods. Transactions on Mathematical Software, 2021.

[6] P. E. Farrell, L. Mitchell, L. R. Scott, and F. Wechsung. A Reynolds-robust preconditioner for the Scott-Vogelius discretization of the stationary incompressible Navier-Stokes equations. The SMAI journal of computational mathematics, 7:75-96, 2021.

[7] V. Girault and P.-A. Raviart. Finite element methods for Navier-Stokes equations. Theory and algorithms, volume 5. Springer, 1986.

[8] J. Guzmán, A. Lischke, and M. Neilan. Exact sequences on Powell-Sabin splits. Calcolo, 57(2):1-25, 2020.

[9] J. Guzman, A. Lischke, and M. Neilan. Exact sequences on Worsey-Farin splits. arXiv preprint arXiv:2008.05431, 2020.

[10] J. Guzmán and M. Neilan. Conforming and divergence-free Stokes elements in three dimensions. IMA Journal of Numerical Analysis, 34(4):1489-1508, 2014.

[11] J. Guzmán and M. Neilan. Inf-sup stable finite elements on barycentric refinements producing divergence-free approximations in arbitrary dimensions. SIAM Journal on Numerical Analysis, 56(5):2826-2844, 2018.

[12] J. Guzmán and L. Scott. The Scott-Vogelius finite elements revisited. Mathematics of Computation, 88(316):515529, 2019.

[13] J. Qin. On the convergence of some low order mixed finite elements for incompressible fluids. PhD thesis, Citeseer, 1994.

[14] J. Qin and S. Zhang. Stability and approximability of the P1-P0 element for Stokes equations. International journal for numerical methods in fluids, 54(5):497-515, 2007.

[15] L. R. Scott and M. Vogelius. Norm estimates for a maximal right inverse of the divergence operator in spaces of piecewise polynomials. RAIRO Modél. Math. Anal. Numér., 19(1):111-143, 1985.

[16] D. Silvester and A. Wathen. Fast iterative solution of stabilised Stokes systems part ii: Using general block preconditioners. SIAM Journal on Numerical Analysis, 31(5):1352-1367, 1994.

[17] U. Trottenberg, C. Ulrich Trottenberg, C. Oosterlee, A. Schuller, A. Brandt, P. Oswald, and K. Stüben. Multigrid. Elsevier Science, 2001.

[18] S. Zhang. A new family of stable mixed finite elements for the 3D Stokes equations. Mathematics of computation, 74(250):543-554, 2005.

[19] S. Zhang. On the P1 Powell-Sabin divergence-free finite element for the Stokes equations. Journal of Computational Mathematics, pages 456-470, 2008

[20] S. Zhang. A family of 3D continuously differentiable finite elements on tetrahedral grids. Appl. Numer. Math., 59(1):219-233, 2009.

[21] S. Zhang. Quadratic divergence-free finite elements on Powell-Sabin tetrahedral grids. Calcolo, 48(3):211-244, 2011.

$\dagger$ Division of Applied Mathematics, Brown University, Providence, Ri 02912, USA

Email address: fabien@brown.edu

$\dagger$ Division of Applied Mathematics, Brown University, Providence, Ri 02912, USA

Email address: johnny_guzman@brown.edu

${ }^{\ddagger}$ Department of Mathematics, University of Pittsburgh

Email address: neilan@pitt.edu

‡Department of Mathematics,University of Pittsburgh

Email address: AMZ56@pitt.edu 